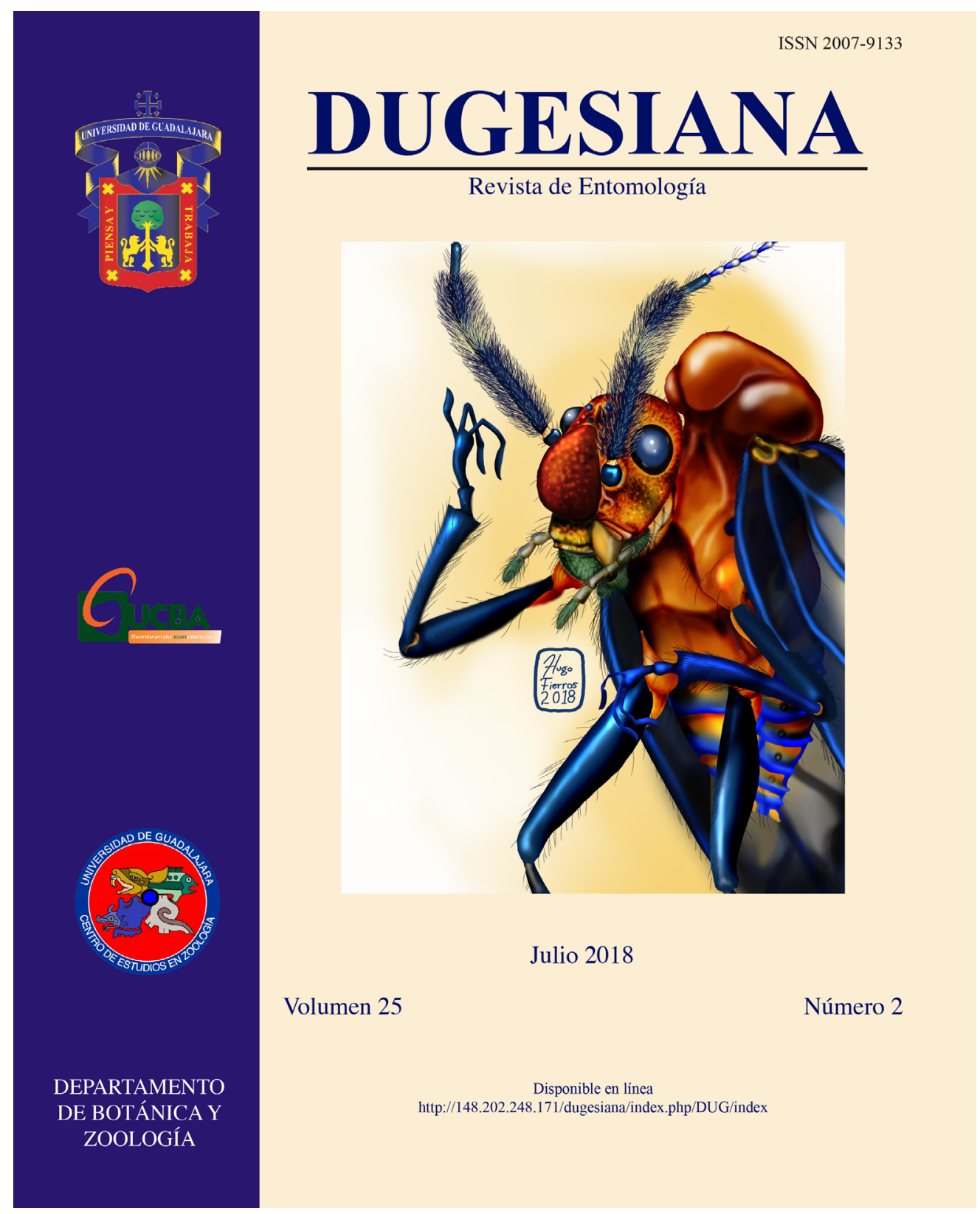

Dugesiana, Año 25, No. 2, julio 2018-diciembre 2018 (segundo semestre de 2018), es una publicación semestral, editada por la Universidad de Guadalajara, a través del Centro de Estudios en Zoología, por el Centro Universitario de Ciencias Biológicas y Agropecuarias. Camino Ramón Padilla Sánchez \# 2100, Nextipac, Zapopan, Jalisco, Tel. 37771150 ext. 33218, http://148.202.248.171/dugesiana/index.php/DUG/index, glenusmx@gmail.com. Editor responsable: José Luis Navarrete Heredia. Reserva de Derechos al Uso Exclusivo 04-2009-062310115100203, ISSN: 2007-9133, otorgados por el Instituto Nacional del Derecho de Autor. Responsable de la última actualización de este número: José Luis Navarrete Heredia, Editor y Ana Laura González-Hernández, Asistente Editorial. Fecha de la última modificación 25 de julio 2018, con un tiraje de un ejemplar.

Las opiniones expresadas por los autores no necesariamente reflejan la postura del editor de la publicación.

Queda estrictamente prohibida la reproducción total o parcial de los contenidos e imágenes de la publicación sin previa autorización de la Universidad de Guadalajara. 


\title{
El género Stenoscelidea en Ecuador, con descripción de dos nuevas especies y nuevos registros (Hemiptera: Heteroptera: Coreidae: Stenoscelideini)
}

\section{The genus Stenoscelidea in Ecuador, with description of two new species and new records (Hemiptera: Heteroptera: Coreidae: Stenoscelideini)}

\author{
Harry Brailovsky ${ }^{1,2}$ y Ernesto Barrera ${ }^{1,3}$ \\ 1,2 Departamento de Zoología, Instituto de Biología, Universidad Nacional Autónoma de México, Apdo Postal \\ 70153, Ciudad de México; coreidae@ib.unam.mx; 1,3 ernesto.barrera@ib.unam.mx
}

\section{RESUMEN}

Se revisa el género Stenoscelidea Westwood, 1842 (Coreidae: Coreinae: Stenoscelideini) para Ecuador, describiendo dos nuevas especies y citando por primera vez a S. blotei Brailovsky y Barrera, 1996 y a S. hilaris Breddin, 1903. Se incluye una clave para separar a las especies, y una serie de fotografías que ilustran el hábito de las mismas, y la peculiar coloración de los segmentos dorsales del abdomen; los parámeros de la mayoría de las especies son incluidos. Se agregan notas biológicas para algunas de las especies.

Palabras clave: Heteroptera, Coreidae, Stenoscelidea, Ecuador, nuevas especies, notas biológicas, clave.

\begin{abstract}
The genus Stenoscelidea Westwood, 1842 (Coreidae: Coreinae: Stenoscelideini) from Ecuador is revised and two new species are described. Stenoscelidea blotei Brailovsky and Barrera, 1996, and S. hilaris Breddin, 1903, are recorded for the first time in Ecuador. A key to the known species is presented. Photographs of the habitus and the unique coloration of the dorsal abdominal segments of these species are presented as well as the parameres of most of them. Notes on the biology of some species are given.
\end{abstract}

Key words: Heteroptera, Coreidae, Stenoscelidea, Ecuador, new species, biological notes, key.

El género Stenoscelidea Westwood, 1842, está integrado por 13 especies todas ellas con una distribución Neotropical a través de Bolivia, Brasil, Colombia, Ecuador, y Perú: S. albovaria Westwood, 1842 (Brasil y Perú), S. atra Brailovsky y Barrera, 1996 (Brasil), S. blotei Brailovsky y Barrera, 1996 (Perú), S. guanaya Brailovsky y Barrera, 2012 (Bolivia), S. hilaris Breddin, 1903 (Bolivia, Colombia y Perú), S. morona Brailovsky y Barrera, 1996 (Ecuador), S. nigroaenea Breddin, 1903 (Bolivia), S. pucalpa Brailovsky y Barrera, 2012 (Perú), S. roraima Brailovsky y Barrera, 1996 (Brazil), S. rubra Montandon, 1895 (Brasil), S. saphes Brailovsky y Barrera, 2012 (Perú), S. tibialis Brailovsky y Barrera, 1996 (Perú) y S. t-nigrum (Costa, 1863) (Brasil) (Packauskas 2010, Brailovsky y Barrera 2012).

El género, redescrito en extenso por Brailovsky y Barrera, 2012, se caracteriza por tener el cuello transversalmente negro; el artejo rostral IV de mayor tamaño, el III de menor tamaño y el I y II subiguales; los callos pronotales elevados y sin puntuaciones; el disco pronotal y escutelar desprovistos de una franja longitudinal media amarilla o anaranjada; las venas coriales no uniformemente amarillas; y tanto las pro-, meso-, y metapleura lisas excepto el margen posterior con finas puntuaciones.

Una sola especie, S. morona, se conocía para Ecuador. Como resultado de este estudio dos nuevas especies son descritas y otras dos, S. blotei y $S$. hilaris, son citadas por primera vez. Se incluye una clave para separar a las mismas.

\section{MATERIALES Y MÉTODOS}

Los acrónimos usados en el texto son: Colección Joe E. Eger (JEEC); Colección Estatal de Artrópodos de Florida, Gainesville, Florida, EUA (FSCA); Museo de Historia Natural de Los Ángeles County, Los Ángeles, California, EUA (LACM); Museo Nacional de Historia Natural, Paris, Francia (MNP); Universidad Autónoma de Chapingo, Chapingo, Estado de México, México (UACH); Instituto de Biología, Universidad Nacional Autónoma de México, México (UNAM); Museo Nacional de Historia Natural, Instituto Smithsoniano, Washington, D. C., EUA (USNM); Universidad Estatal de Utah, Logan, Utah, EUA (USUL).

\section{RESULTADOS Y DISCUSIÓN}

Stenoscelidea cibriana Brailovsky \& Barrera n. sp. (Figuras 1, 9, 14, 18, 22-23)

http://zoobank.org/707303D0-8FD8-4CA4-9255C613B7E3F40A

Holotipo macho: Ecuador, Provincia Napo, cerca de Puerto Misahuali, 1650-1900' pies sobre el nivel del mar, 1²'4.2”S, 77³9'49.2”W, 6-19/IX/1998. Recolectado utilizando pescado en descomposición y colocado en trampas para mariposas. Col. J. E. Eger. Depositado en UNAM. Paratipos. Tres machos y 13 hembras con los mismos datos que el holotipo. Depositados en FSCA, UNAM y USNM. 1 hembra: Ecuador, Provincia Napo, Misahuali cerca de Tena, 26/VIII al 2/IX/2000. Recolectado en trampa de luz 
negra. Col. Steven and Paul Keller. Depositado en USUL. 1 macho: Ecuador, Provincia Pastaza, Tzapino, $32 \mathrm{~km}$ NE Tigueno, $400 \mathrm{~m}, 1^{\circ} 11^{\prime} \mathrm{S}, 77^{\circ} 14^{\prime} \mathrm{W}, 26 / \mathrm{V} / 1976$. Col. J. Cohen. Depositado en USNM. 1 macho: Ecuador, Napo, Yasuni N. P. $220 \mathrm{~m}, 0.67^{\circ} \mathrm{S}, 18.26^{\circ} \mathrm{W}, 18-26 / \mathrm{V} / 1996$. Recolectado utilizando luz ultravioleta. Col. B. Brown, J. Cantley y P. Hibbs. Depositado en LACM. 1 hembra: Ecuador, Dayuma, 7/IV/2012. Col. K. Todaka. Depositado en UNAM.

Holotipo macho. Coloración dorsal. Cabeza amarilla, con el cuello, los tubérculos ocelares y dos franjas longitudinales que corren laterales a la línea media desde el cuello hasta la base del tylus de color negro; tylus negro; artejo antenal I anaranjado pálido con una corta franja basal externa negra; artejos II y III anaranjado pálido y el IV negro o pardo obscuro; tubérculo postocular amarillo y con la cara anexa al borde inferior de los ojos pardo obscuro; pronoto incluyendo el collar y los callos amarillos, con las siguientes áreas de color negro: bordes anterolaterales, ángulos humerales, márgenes posterolaterales y una mancha en " $T$ " adornando el disco pronotal; margen posterior amarillo y con el tercio medio negro; escutelo amarillo con una franja longitudinal negra en el tercio basal medio; clavus negro con el tercio distal anaranjado pálido; vena claval anaranjado obscuro; corium negro con el borde costal, el ángulo apical, el margen apical y una franja longitudinal que corre a los lados del clavus anaranjado pálido; membrana hemelitral negra; segmentos conexivales III-VII amarillos con el tercio posterior negro; segmentos dorsales del abdomen IIIVI con una franja longitudinal amarilla continua y lateralmente delimitada por un banda negra incompleta al tener el margen posterior amarillo; segmento abdominal VII negro con una mancha triangular amarilla en el tercio basal medio (Fig. 14). Coloración ventral. Cabeza incluyendo los artejos rostrales I-IV amarillos, con la superficie mesial, el tylus, el tercio inferior de los tubérculos anteníferos, dos manchas cortas por debajo de los jugum, y el ápice del artejo rostral IV negro; prosternón amarillo, con dos manchas basales laterales a la línea media negros; propleura amarilla con una amplia mancha negra en "8"; mesosternón amarillo y lateralmente delimitado por dos franjas negras longitudinales completas; mesopleura amarilla con dos amplias manchas negras en "C" invertidas; metasternón amarillo; metapleura amarilla con dos amplias manchas negras que no llegan a fusionarse y que dejan entre ellas un espacio amarillo; acetábulos de los tres pares de patas amarillos; lóbulo anterior y posterior del peritremo osteolar metatorácico negro; pata anterior y media anaranjado pálido, excepto las coxas que poseen una franja transversal anterior negra; pata posterior con las coxas y trocánteres anaranjado pálido; fémur anaranjado pálido y con el borde apical pardo obscuro; tibia anaranjado pálido con las dilataciones negras; dilatación externa con una mancha discoidal amarilla; tarsos anaranjado pálido. Abdomen. Esternito abdominal III amarillo y con cuatro manchas cercanamente circulares negras; esternitos IV y $\mathrm{V}$ amarillos con el margen superior y cuatro manchas cercanamente circulares negras; esternito VI negro con el margen inferior y una franja mesial completa e irregular que corre longitudinalmente a través del esternito amarillo; esternito VII amarillo con el margen inferior, una mancha longitudinal delgada que cruza el tercio medio del esternito y dos manchas triangulares laterales a la línea media amarillos; margen pleural de los esternitos abdominales III-VII incluyendo los espiráculos amarillos y con el tercio posterior negro; pigóforo negro con el margen posteroventral amarillo.

Estructura. Cabeza. Rostro alcanzando el tercio medio del metasternón. Pronoto. Collar evidente; ángulos frontales redondeados; ángulos humerales redondeados, no expuestos. Patas. Fémures de los tres pares de patas armados ventralmente con una hilera de 3 o 4 espinas, cortas y aguzadas; tibia posterior con la dilatación externa ampliamente ensanchada y con una escotadura terminal; dilatación interna lanceolada, medianamente dilatada y continua (Fig. 1). Pigóforo. Margen posteroventral con una amplia concavidad mesial (Fig. 18). Parámeros. Ver figuras 22-23.

Hembra. Hábitos y coloración semejante al holotipo macho. Segmentos dorsales del abdomen VIII y IX negros; segmento conexival VIII negro con el ángulo anterior amarillo; segmento conexival IX negro; gonocoxa I negra; paratergitos VIII y IX amarillos.

Biología. Se recolectaron en trampas para mariposas donde se incluía como cebo a pescados en descomposición. También fueron atraídos a la luz utilizando trampas tanto de luz ultravioleta como de luz negra.

Medidas (mm). Holotipo macho. Longitud total del cuerpo: 11.90. Cabeza: longitud total: 1.36; anchura a través de los ojos: 2.30; espacio interocular: 1.06; espacio interocelar: 0.41; longitud de los artejos antenales: I, 3.00, II, 3.85, III, 3.10; IV, 6.40. Pronoto. Longitud total: 2.55; anchura a través de los ángulos humerales: 3.25. Escutelo. Longitud total: 1.80; anchura máxima: 1.25.

Medidas (mm). Paratipo hembra. Longitud total del cuerpo: 14.43. Cabeza: longitud total: 1.40; anchura a través de los ojos: 2.40; espacio interocular: 1.06; espacio interocelar: 0.41 ; longitud de los artejos antenales: I, 3.05, II, 3.75, III, 3.20; IV, 6.20. Pronoto. Longitud total: 2.90; anchura a través de los ángulos humerales: 4.00. Escutelo. Longitud total: 2.00; anchura máxima: 1.60.

Etimología. Nos complace dedicar esta especie al Ingeniero Agrónomo David Cibrián Tovar como un reconocimiento a sus estudios ligados a los insectos forestales de la República Mexicana.

Discusión. Afín a S. t-nigrum (Costa) 1863, por guardar un patrón de coloración similar en el disco pronotal donde la " $T$ " negra resalta sobre un fondo amarillo; el clavus negro con el tercio apical y en ocasiones la vena claval anaranjada obscura o amarilla; el tylus negro; el artejo antenal I anaranjado con una corta franja basal negra; las patas anteriores y medias anaranjado pálido; y el escutelo amarillo con una mancha discoidal basal negra. En $S$. cibriana Brailovsky y Barrera n. sp., los artejos antenales II 
y III son anaranjado pálido; el fémur posterior anaranjado pálido con el borde apical y eventualmente el tercio apical negro; la tibia posterior con la unión basal negra; la dilatación externa de la tibia posterior conspicuamente ensanchada (Fig. 1); y los segmentos dorsales del abdomen III-VI con una franja longitudinal amarilla continua delimitada lateralmente por una banda negra incompleta al tener el margen posterior amarillo (Fig. 14). En $S$. t-nigrum los artejos antenales II y III son pardo rojizos o pardo anaranjados; el fémur posterior con el borde apical o el tercio apical anaranjado pálido; la tibia posterior con la unión basal anaranjado pálido; la dilatación externa de la tibia posterior medianamente expuesta; y los segmentos dorsales del abdomen III-VI adornados en su línea media con una mancha cuadrada amarilla, delimitada en su cara anterior y posterior por una franja negra y lateralmente delimitada por dos franjas negras ensanchadas (Figs. 1315).

Stenoscelidea naevilla Brailovsky \& Barrera n. sp. (Figuras 7, 12, 16)

http://zoobank.org/31983839-9DE3-4919-BD604787BA8C0C43

Holotipo hembra: Ecuador, Napo, Tiputini, Biodiversity Station, $216 \mathrm{~m}, 0^{\circ} 37^{\prime} 55^{\prime} \mathrm{S}, 7^{\circ} 08^{\prime} 39^{\prime} \mathrm{W}, 4 / \mathrm{VII} / 1998$. Recolectado mediante fumigación con insecticida de hojas verdes, limpias en su mayoría y algunas con líquenes y briofitas creciendo sobre ellas. Col. T. L. Erwin, et al. Depositado en USNM. Paratipo. Una hembra. Perú, Loreto, Iquitos, ExplorNapo, $3.25723^{\circ} \mathrm{S}, 72.90943^{\circ} \mathrm{W}, 30 / \mathrm{VI} / 2015$. Recolectado utilizando trampas para la captura de la mosca de la fruta con cebo mixto de putrecina y acetato de amonio. Col. U. Cabral. Depositado en UNAM.

Holotipo hembra. Coloración dorsal. Cabeza amarilla con dos manchas negras laterales a la línea media y cercanas a los tubérculos anteníferos; cuello negro; artejos antenales I-IV anaranjado pálido; pronoto amarillo con las siguientes áreas de color negro: bordes anterolaterales, una franja transversal que recorre el margen posterior (el borde posterior se conserva amarillo), y una mancha en "T" adornando el disco pronotal; escutelo amarillo; clavus negro; corium tricoloro, con el ángulo apical crema, el margen costal y el endocorium cercano al tercio apical negro y el resto del endocorium basal y medio amarillo; membrana hemelitral pardo obscuro; conexivo amarillo y con el tercio posterior pardo; segmentos dorsales del abdomen III-VI amarillos y delimitado en su margen anterior y posterior por una franja transversal negra; segmentos dorsales VII-IX negros (Fig. 16). Coloración ventral. Cabeza amarilla con una mancha negra mesial; artejos rostrales I-IV amarillos; prosternón y mesosternón amarillos; metasternón amarillo y delimitado a cada lado por una franja negra brillante; propleura amarilla y con dos manchas negras, una en forma "8" que recorre transversalmente el segmento y la otra delgada, con trayectoria longitudinal que recorre el borde prosternal; mesopleura y metapleura amarillas y con una franja transversal irregular negra que corre a través de los mismos; patas amarillas excepto las coxas de los tres pares de patas en donde la mitad anterior es negra brillante y la mitad posterior amarilla; tibia posterior amarilla con la dilatación externa e interna negras y provistas cada una de ellas con un disco central amarillo; acetábulos de los tres pares de patas amarillos; lóbulo anterior y posterior del peritremo osteolar metatorácico negro. Abdomen. Esternitos abdominales IIIVI en su tercio medio amarillos con una mancha negra e irregular que recorre la mitad del mismo; lateralmente con dos franjas negras que delimitan el manchado amarillo; margen pleural incluyendo los espiráculos amarillos y con los ángulos anterior y posterior negros; esternito abdominal VII negro con el borde posterior amarillo; margen pleural incluyendo los espiráculos amarillos y con el ángulo anterior y el tercio posterior negros; gonocoxa I negra; paratergitos VIII y IX amarillos.

Estructura. Cabeza. Rostro alcanzando el margen posterior del metasternón. Pronoto. Collar evidente; ángulos frontales redondeados; ángulos humerales no expuestos y con el ápice subagudo. Patas. Fémures de los tres pares de patas armados ventralmente con dos espinas subapicales cortas y agudas y una hilera de 4 a 7 espinas alineadas detrás de las anteriores; tibia posterior con la dilatación externa conspicuamente expandida, filiforme, con los bordes enteros y con una escotadura terminal; dilatación interna lanceolada y medianamente expandida (Fig. 7).

Variación. 1, cabeza dorsalmente amarilla con el cuello y dos franjas longitudinales negras que corren a los lados de la línea media desde los ocelos hasta los jugum. 2, disco pronotal con los brazos negros de la "T" tocando los márgenes anterolaterales. 3 , fémur posterior amarillo con el tercio apical negro. 4, segmentos dorsales VIII y IX del abdomen negros con el borde superior amarillo. 5, margen pleural del abdomen enteramente amarillo.

Macho. Desconocido.

Biología. El ejemplar ecuatoriano fue capturado mediante fumigación con insecticida de hojas verdes, limpias en su mayoría, y algunas con líquenes y briofitas creciendo sobre ellas, en tanto que el ejemplar peruano fue recolectado en trampas para capturar la mosca de la fruta con cebo mixto de putrecina y acetato de amonio.

Medidas (mm). Holotipo hembra. Longitud total del cuerpo: 14.50. Cabeza: longitud total: 1.35; anchura a través de los ojos: 2.30; espacio interocular: 1.15 ; espacio interocelar: 0.49; longitud de los artejos antenales: I, 2.85, II, 3.75, III, 3.00; IV, 6.50. Pronoto. Longitud total: 3.05; anchura a través de los ángulos humerales: 4.05. Escutelo. Longitud total: 2.15; anchura máxima: 1.80 .

Etimología. Del Latin “naevus" (= marca) en referencia a la franja transversal negra que cruza el margen posterior del disco pronotal.

Discusión. Stenoscelidea naevilla Brailovsky \& Barrera n. sp., al igual que $S$. hilaris Breddin 1903, poseen los artejos antenales I-IV y las patas anteriores y medias 
de color anaranjado o amarillo pálido (Figs. 10, 12). En $S$. naevilla el margen posterior del disco pronotal tiene una franja transversal continua de color negro la cual cruza a través de los ángulos humerales, dejando el borde posterior amarillo; el clavus es enteramente negro; el fémur posterior es anaranjado con el tercio apical negro; la dilatación externa de la tibia posterior es filiforme y conspicuamente dilatada (Fig. 7); y los segmentos dorsales del abdomen III-VI son amarillos y solo el borde anterior y posterior pardos (Fig. 16). En S. hilaris el margen posterior del disco pronotal carece de una franja transversal continua de color negro; el clavus es negro con el tercio apical y la vena claval anaranjada o amarillo obscura; el fémur posterior enteramente anaranjado o amarillo; la dilatación externa de la tibia posterior del macho escasamente expuesta (Fig. 4) y en la hembra medianamente dilatada (Fig. 3) y los segmentos dorsales del abdomen III-VI poseen en su línea media una mancha cuadrada amarilla, delimitada en su cara anterior y posterior por una franja negra y lateralmente delimitada por dos franjas negras ensanchadas (Fig. 15).

\section{Stenoscelidea blotei Brailovsky y Barrera}

(Figuras 5-6, 8, 13, 17, 20-21)

Stenoscelidea blotei Brailovsky y Barrera 1996: 108-114

Diagnosis. En esta especie los artejos antenales I-III son amarillos y el IV castaño rojizo; las patas anterior y medias anaranjadas; y los segmentos dorsales del abdomen III-VI poseen en su línea media una mancha cuadrada amarilla, delimitada en su cara anterior y posterior por una franja negra y lateralmente delimitada por dos franjas negras ensanchadas (Fig. 13). Se reconoce por tener el fémur posterior negro excepto la unión basal amarilla o anaranjado rojiza; el escutelo negro con los márgenes laterales y el ápice amarillos; la cabeza dorsal y ventralmente negra al igual que la pro-, meso-, y metapleura. El disco pronotal es negro y eventualmente con un manchado irregular amarillo. La dilatación externa de la tibia posterior de la hembra está ligeramente expuesta y la interna lanceolada y menos pronunciada (Fig. 5) y en el macho la dilatación externa está reducida a una tenue expansión y la interna es difícil de discernir (Fig. 6).

Brailovsky y Barrera (1996) al describir a esta especia señalaron que la dilatación externa de la tibia posterior de la hembra era lanceolada. Al examinar la serie aquí estudiada rectificamos la misma señalando que la dilatación externa al igual que en el macho es reducida y poco expuesta (Figs. 5-6).

Distribución. Solo conocida de la localidad tipo: Perú (Callanga) (Brailovsky y Barrera 1996, Packauskas 2010).

Nuevos registros. 9 machos, 14 hembras: Ecuador, Provincia Napo, cerca de Puerto Misahuali, 1650-1900' pies sobre el nivel del mar, $1^{\circ} 2$ '4.2"S, $77^{\circ} 39^{\prime} 49.2^{\prime \prime} \mathrm{W}$, 6-19/IX/1998. Recolectado utilizando pescado en descomposición y colocado en trampas para mariposas. Col. J. E. Eger. Depositado en FSCA, UNAM y USNM. 2 machos, 1 hembra: Ecuador, Napo, Misahuali, 26/VIII/ al 2/IX/2000. Col. S. y P. Keller. Depositado en USUL. 1 hembra: Ecuador, Napo, Misahuali, cerca Tena, 6-19/ X/2001. Col. C. Brammer. Depositado en USUL.

\section{Stenoscelidea hilaris Breddin}

(Figuras 4, 10, 15, 19, 24)

Stenoscelidea hilaris Breddin 1903: 376

Diagnosis. Esta especie queda segregada por tener los artejos antenales I-IV, el escutelo y las patas anterior y media amarillas; la cabeza dorsalmente amarilla con los tubérculos ocelares y dos mancha basales cercanas al tylus y dispuestas a los lados de la línea media negras; la tibia posterior de la hembra con la dilatación externa medianamente expuesta y con una escotadura terminal y la interna escasamente expuesta (Fig. 3) y en el macho tanto la dilatación externa como interna son imperceptibles (Fig. 4); y los segmentos dorsales del abdomen III-VI poseen en su línea media una mancha cuadrada amarilla, delimitada en su cara anterior y posterior por una franja negra y lateralmente delimitada por dos franjas negras ensanchadas (Fig. 15).

Distribución. Originalmente descrita de Bolivia, sin mencionar una localidad concreta y posteriormente citada para Colombia y Perú (Breddin 1903, Blöte 1938, Brailovsky y Barrera 1996, Packauskas 2010).

Nuevos registros. 3 machos, 10 hembras: Ecuador, Provincia Napo, cerca de Puerto Misahuali, 1650-1900' pies sobre el nivel del mar, $1^{\circ} 2{ }^{\prime} 4.2^{\prime \prime} \mathrm{S}, 7^{\circ} 39^{\prime} 49.2^{\prime \prime} \mathrm{W}$, 6-19/IX/1998. Recolectado utilizando pescado en descomposición y colocado en trampas para mariposas. Col. J. E. Eger. Depositado en FSCA, UNAM y USNM. 3 hembras: Ecuador, Provincia Napo, cerca de Puerto Misahuali, $1^{\circ}{ }^{\prime}$ 'S, $77^{\circ} 40^{\prime} \mathrm{W}, 26 / \mathrm{VIII} /$ al 2/IX/2000, y 6-19/ X/2001. Col. S. y P. Keller y C. Brammer. Depositados en USUL.

\section{Stenoscelidea morona Brailovsky y Barrera}

(Figuras 2, 11)

Stenoscelidea morona Brailovsky y Barrera 1996: 114-116

Diagnosis. Esta atractiva especie se caracteriza por tener los artejos antenales I-IV y la unión basal de la tibia posterior negra; el escutelo amarillo con una mancha negra discoidal o alargada en el tercio basal medio; y los segmentos dorsales del abdomen III-VI poseen en su línea media una mancha cuadrada amarilla, delimitada en su cara anterior y posterior por una franja negra y lateralmente delimitada por dos franjas negras ensanchadas. La tibia posterior de la hembra con la dilatación externa convexa y expuesta y la dilatación interna lanceolada (Fig. 2) (el macho se desconoce).

Distribución. Solo se conoce del Ecuador a través de la descripción original donde se le cita para Pastaza, en el Rio Macuma a $10 \mathrm{~km}$ del Rio Morona (Brailovsky y Barrera 1996, Packauskas 2010).

Nuevos registros. 1 hembra: Ecuador, Tungamanga, 29 km E de Baños, 5130' sobre el nivel del mar, 25/I/1976. 
Col. Spangler et al. Depositado en USNM.

\section{Clave para separar las especies ecuatorianas de Stenoscelidea}

1. Artejos antenales I-III negros S. morona Brailovsky y Barrera, 1996 (Fig. 11)

1' Artejos antenales I-III amarillos o anaranjado pálido

2. Escutelo negro con los márgenes laterales y el ápice amarillos; fémur posterior negro con la unión basal amarilla o anaranjado rojiza; cabeza dorsal y ventralmente negra; pro-, meso-, y metapleura negra

S. blotei Brailovsky y Barrera, 1996 (Figs. 8, 13)

2' Escutelo amarillo y con o sin una mancha negra discoidal o alargada en el tercio basal medio; fémur posterior amarillo o anaranjado y con o sin la unión apical negra; cabeza dorsal y ventralmente mayormente amarilla; pro-, meso-, y metapleura amarilla y con manchas irregulares negras.... 3 3. Margen posterior del disco pronotal con una franja transversal negra continua que cruza a través de los ángulos humerales, dejando el borde posterior amarillo; segmentos dorsales del abdomen III-VI amarillos y solo con el borde anterior y posterior pardos (Fig. 16); clavus enteramente negro ...... S. naevilla Brailovsky y Barrera n. sp. (Fig. 12) 3' Margen posterior del disco pronotal desprovisto de una franja transversal negra continua y con el borde posterior no enteramente amarillo; segmentos dorsales del abdomen con otra condición (Figs. 13-15); clavus no enteramente negro .. 4 4. Segmentos dorsales del abdomen III-VI con una franja longitudinal amarilla continua, lateralmente delimitada por una franja longitudinal negra incompleta al tener el margen posterior amarillo (Fig. 14); tibia posterior de la hembra con la dilatación externa ampliamente ensanchada y con una escotadura terminal (Fig.1).

S. cibriana Brailovsky y Barrera n. sp. (Figs. 9, 14) 4' Segmentos dorsales del abdomen III-VI en su línea media están adornados con una mancha amarilla cuadrada la cual está delimitada en su cara anterior y media por una banda negra y lateralmente delimitada por dos franjas negras ensanchadas (Fig. 15); tibia posterior de la hembra medianamente expuesta y con una escotadura terminal (Fig. 3) . S. hilaris Breddin (Figs. 10, 15)

\section{AGRADECIMIENTOS}

Esta contribución está dedicada al Ingeniero Agrónomo David Cibrián Tovar (UACH) por su destacada labor académica y científica enfocada al conocimiento de los insectos forestales. Así también agradecemos a las siguientes personas e instituciones por el préstamo del material que sirvió de base para este estudio: Joe E. Eger (JEEC), Susan Halbert (FSCA), Brian Harris (LACM), Thomas J. Henry (USNM), y Wilford J. Hanson (USUL). Especial agradecimiento a Dominique Pluot (MNP) por el envío de la literatura solicitada y a Julio Cesar Montero y Diana Martínez Almaguer (UNAM) por el diseño de las placas incluidas en el texto. Oscar Federico Francke Balle (UNAM) por la revisión crítica del manuscrito.

\section{LITERATURA CITADA}

Blöte, C. H. 1938. Catalogue of the Coreidae in the Rijksmuseum van Naturlijke Histoire. Part IV. Coreinae, Third Part. Zoologische Mededeelingen (Leiden), 20: 275-308.

Brailovsky, H., y E. Barrera. 1996. Cinco especies nuevas del género Stenoscelidea (Hemiptera: Heteroptera: Coreidae: Acanthocephalini). Anales del Instituto de Biología, Universidad Nacional Autónoma de México, Serie Zoología, 67 (1): 103-121.

Brailovsky, H. y E. Barrera. 2012. Review of the Stenoscelidea complex and key to the known genera of Stenoscelideini (Hemiptera: Heteroptera: Coreidae). Acta Entomologica Musei Nationalis Pragae, 52 (2): 355-370.

Breddin, G. 1903. Beitrage zur Hemipteres fauna der Anden. Sitzungsberichte der Gesellschaft Naturforschender Freunde zu Berlin, 8: 366-383.

Packauskas, R. 2010. Catalog of Coreidae, or Leaf-Footed Bugs of the New World. Fort Hays Studies, Fourth Series, 5: 1-270.

Westwood, J. O. 1842. A catalogue of Hemiptera in the collection of the Rev. F. W. Hope, with short Latin description of new species. Part II. J. C. Bridgewater, London.

Recibido: 17 de abril 2018

Aceptado: 16 de mayo 2018 


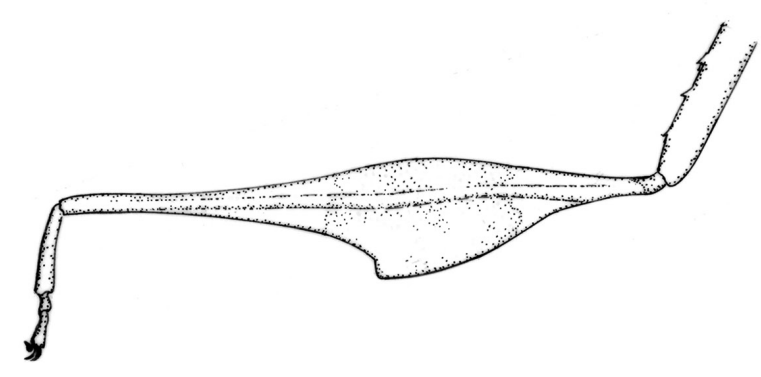

1

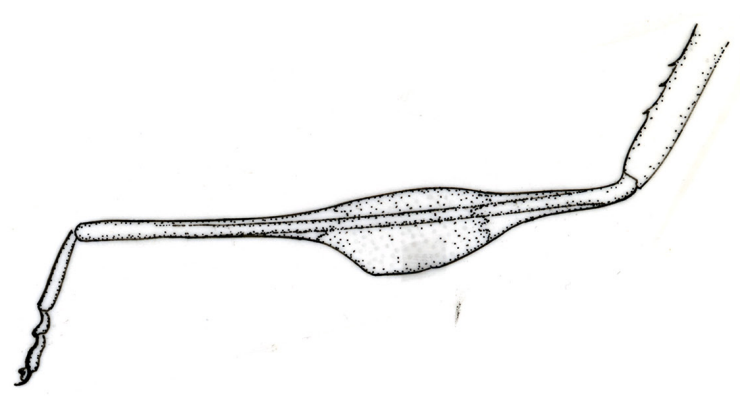

3

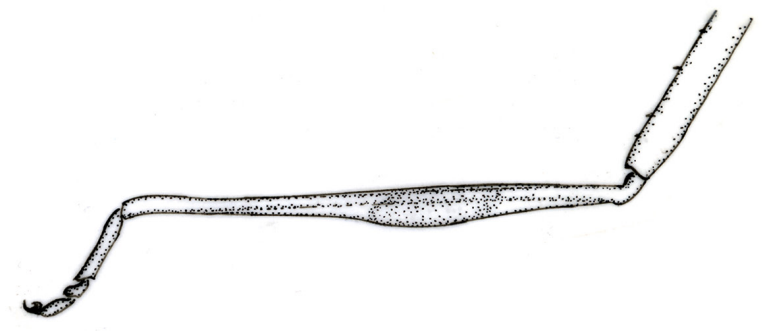

5

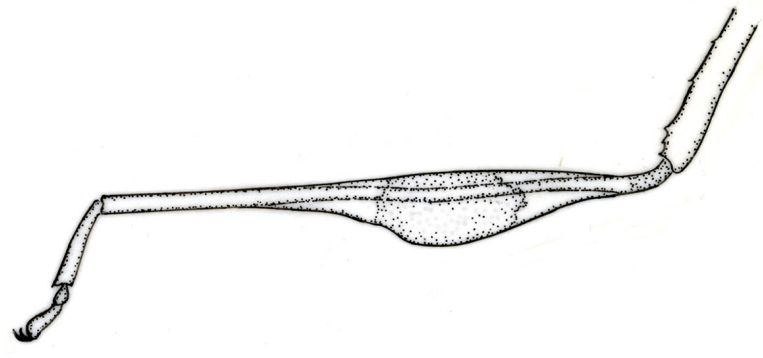

2

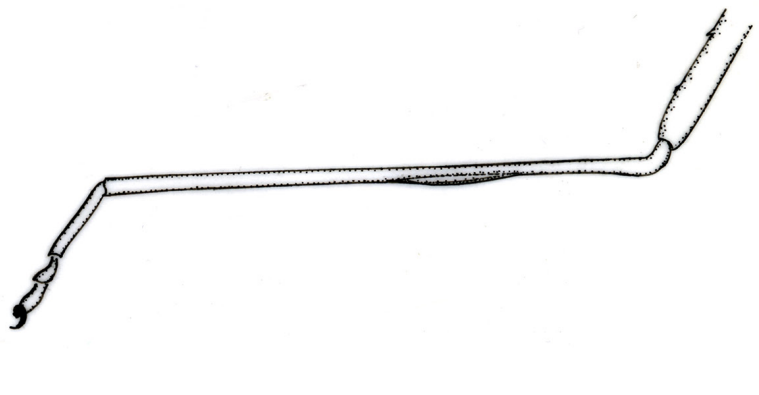

4

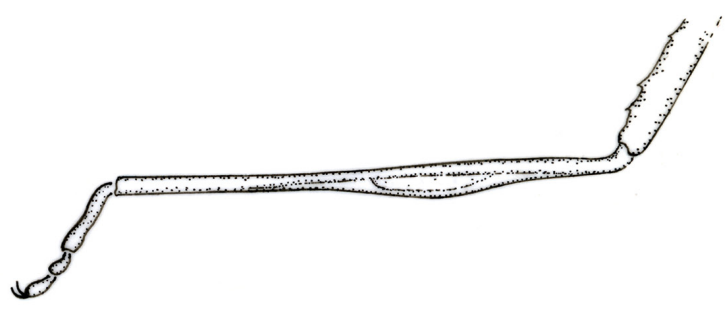

6

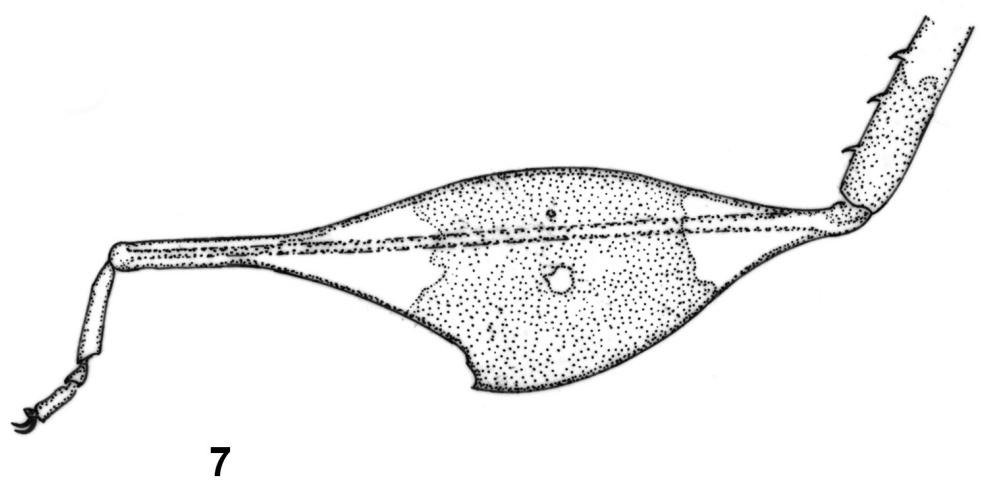

Figuras 1-7. Tibia posterior de Stenoscelidea spp. 1. S. cibriana n. sp. (hembra). 2. S. morona Brailovsky y Barrera, 1996 (hembra). 3-4. S. hilaris Breddin, 1903. 3. Hembra. 4. Macho. 5-6. S. blotei Brailovsky y Barrera, 1996. 5. Hembra. 6. Macho. 7. S. naevilla n. sp. (hembra). 


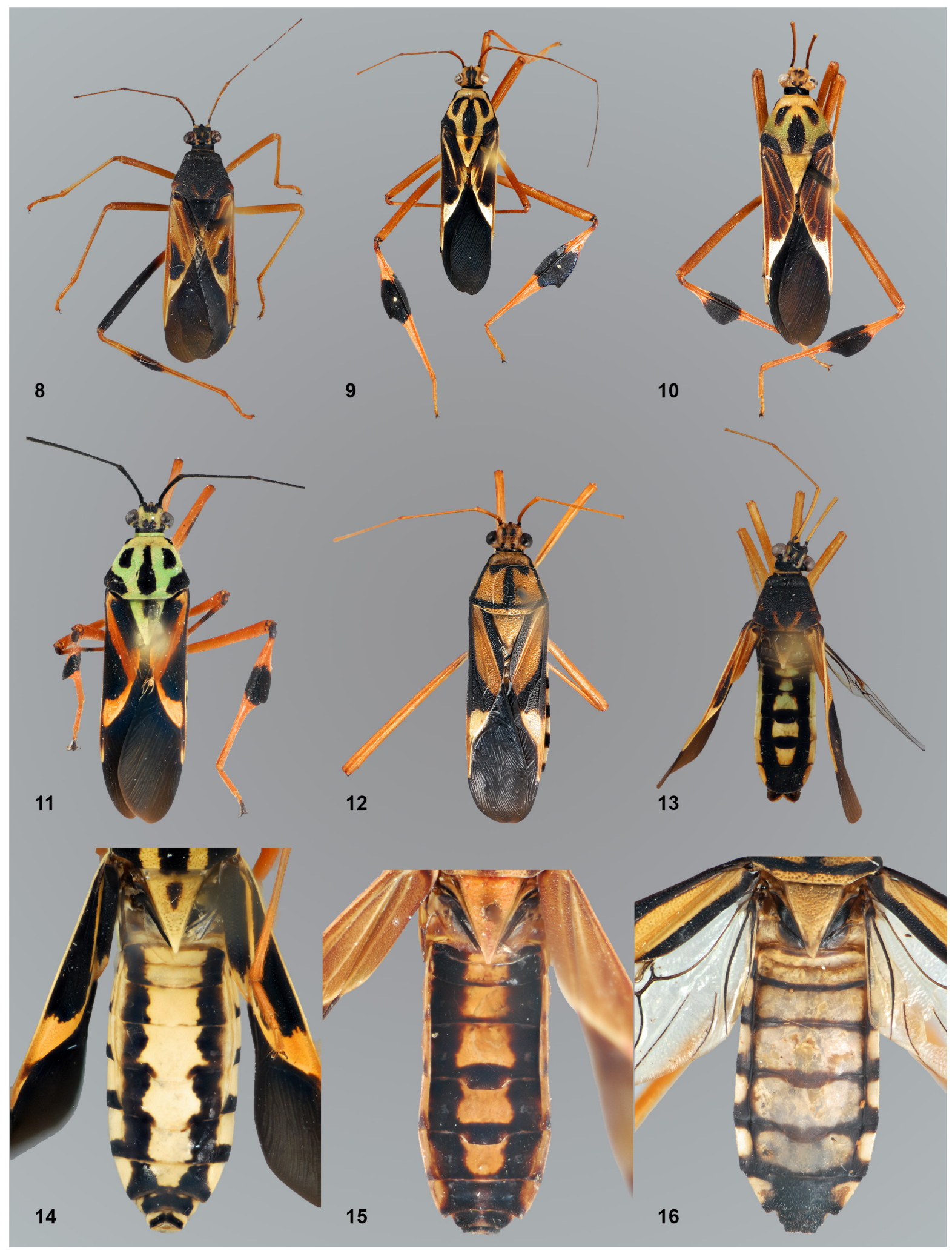

Figuras 8-12. Vista dorsal de Stenoscelidea spp. 8. S. blotei Brailovsky y Barrera, 1996 (hembra). 9. S. cibriana n. sp. (hembra). 10. S. hilaris Breddin, 1903 (hembra). 11. S. morona Brailovsky y Barrera, 1996 (hembra). 12. S. naevilla n. sp. (hembra). Figuras 13-16. Segmentos dorsales del abdomen de Stenoscelidea spp. 13. S. blotei Brailovsky y Barrera, 1996 (macho). 14. S. cibriana n. sp. (hembra). 15. S. hilaris Breddin, 1903 (hembra). 16. S. naevilla n. sp. (hembra). 


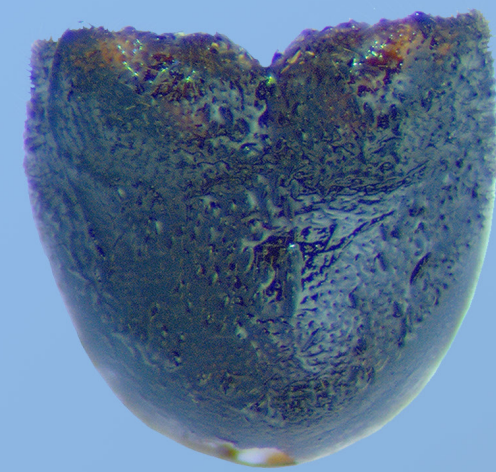

17

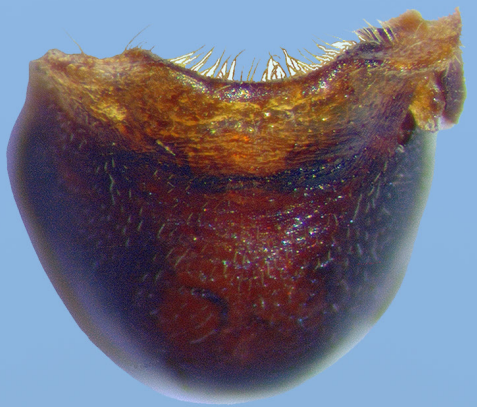

18

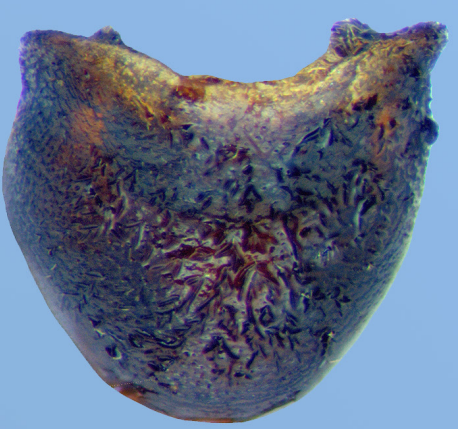

19

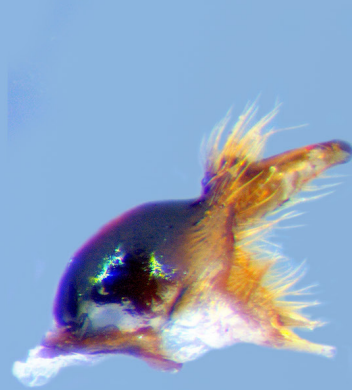

20

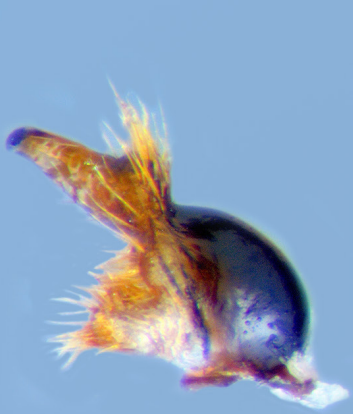

21

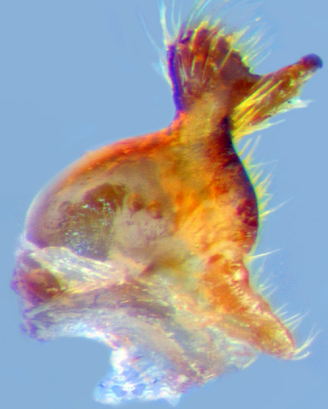

22

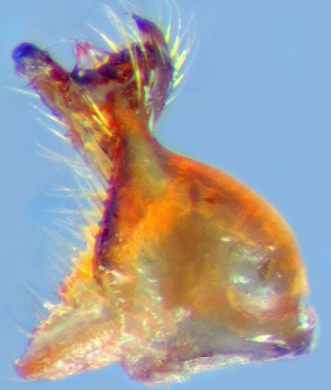

23

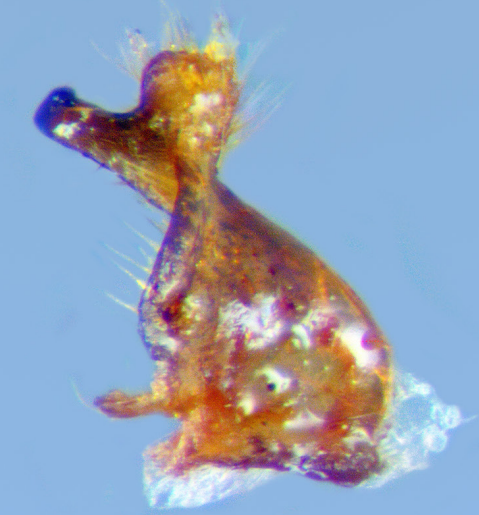

24

Figuras 17-19. Capsula genital del macho de Stenoscelidea spp. 17. S. blotei Brailovsky y Barrera, 1996. 18. S. cibriana n. sp. 19. S. hilaris Breddin, 1903. Figuras 20-24. Parámeros de Stenoscelidea spp. 20-21. S. blotei Brailovsky y Barrera, 1996. 22-23. S. cibriana n. sp. 24. S. hilaris Breddin, 1903. 